\title{
El proceso de Convergencia Europea y el periodismo especializado: el caso del Periodismo Político
}

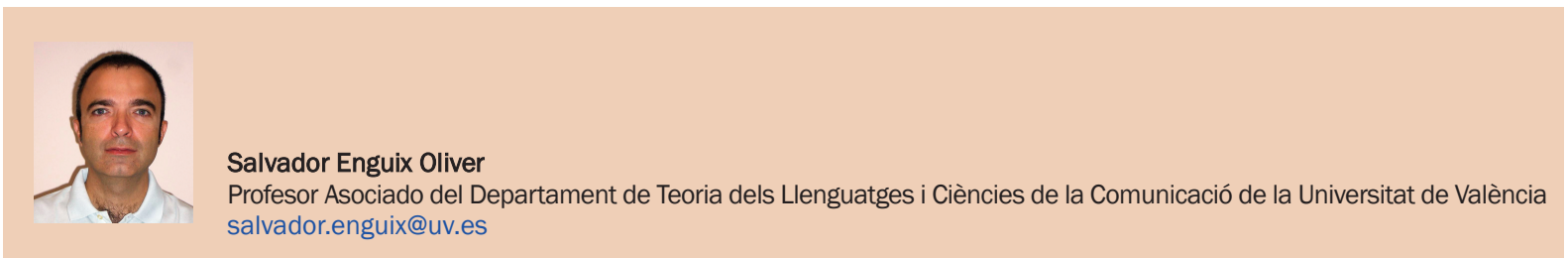

|Fecha presentación: 26/03/2013 | Aceptación: 30/05/2013 |Publicación: 21/06/2013

Resumen

En este trabajo analizamos los planes de Grado en Periodismo resultantes del proceso de adaptación al EEES, prestando especial atención a la inclusión de materias de Periodismo Político. Concluimos que los perfiles profesionales identificados en el Libro Blanco sobre los títulos de grado en comunicación han condicionado unos planes formativos que en general no otorgan suficiente importancia a las materias de Periodismo Especializado y, muy especialmente, al Periodismo Político, ámbito al que se adscribe la mayoría de los contenidos de la prensa escrita. Así, sólo 13 de los 37 grados incluyen formación específica en Periodismo Político. Esta situación revela una divergencia entre la realidad profesional y la formación universitaria que debería ser subsanada en el futuro.

Palabras clave: periodismo, periodismo político, grados europeos, espacio europeo de educación superior, perfiles profesionales

\begin{abstract}
Resum
En aquest treball analitzem els plans de Grau en Periodisme resultants del procés d'adaptació a l'EEES, amb especial atenció a la inclusió de matèries de Periodisme Polític. Concloem que els perfils professionals identificats al Llibre Blanc, i reproduïts quasi literalment en molts d'aquests plans de grau, han condicionat uns plans formatius que en general no atorguen suficient importància a les matèries de Periodisme Especialitzat i, molt especialment, al Periodisme Polític, àmbit al que s'adscriu la majoria dels continguts de la premsa escrita. Així, només 13 dels 37 graus inclouen formació específica en Periodisme Polític. Aquesta situació evidencia una divergència entre la realitat professional i la formació universitària que hauria de ser resolta en el futur.
\end{abstract}

Paraules clau: periodisme, periodisme polític, graus europeus, espai europeu d'educació superior, perfils professionals

\begin{abstract}
This paper analyze the degree in Journalism plans resulting from the adaptation to the EAHE, with particular attention to the inclusion of Political Journalism topics. The profiles identified in the White Paper about degrees in communication, and reproduced in many of these degree plans, have conditioned some training plans that generally do not give enough importance to Specialized Journalism and, especially, Political Journalism, area to which ascribes most of the contents of the press. Thus, only 13 of the 37 degrees include specific courses in Political Journalism. This situation reveals a divergence between professional reality and university training that should be solved in the future.
\end{abstract}

Key words: journalism, political journalism, european degrees, european area of higher education, professional profiles. 


\section{Introducción}

Las facultades de periodismo españolas, casi todas creadas desde la conclusión de la Transición y algunas muy recientemente, surgen por una necesidad evidente de formar profesionales en un periodo, entre 1982 y 1996, de enorme expansión de los medios de comunicación de masas: aparición de televisiones privadas y autonómicas, nuevos periódicos e incluso delegaciones territoriales de los ya existentes, muchos gabinetes de prensa de muchas instituciones nuevas, etcétera. Esas facultades, y la teoría clásica sobre la que se sustentaron, formalizaron programas basados en una visión del periodismo propia del siglo XX (en la prensa, por cierto, con el retrovisor puesto en el XIX), cuando no existía Internet, ni los diarios digitales, ni los buscadores on line, ni las redes sociales, ni nada de lo que ahora condiciona, inevitablemente, la profesión periodística.

Todo esto es cierto, y lo es aún más que las facultades de periodismo están sufriendo en igual medida que los medios de masas convencionales: la revolución digital es tan acelerada que difícilmente una estructura de anclaje medieval, como es la de las universidades, puede adaptarse al mismo ritmo para ofrecer contenidos que respondan a las nuevas necesidades, a las nuevas habilidades que se exigen a los periodistas. Ni siquiera los propios periódicos saben bien cómo actuar ante tantos nuevos retos, y cada gran diario del mundo está adoptando su propio modelo (Kawamoto 2003; Fogel y Patiño 2005; Jones y Salter 2012); el modelo que ofrece Internet es tan completo - permite combinar todos los lenguajes de la comunicación - que aún no hemos acotado todas sus posibilidades y, menos aún, el modelo de negocio, que es el asunto que amenaza la supervivencia, principalmente, de los medios escritos.

A esta situación de crisis interna de la profesión y la industria periodística se ha sumado, en la última década, la necesidad de transformar los planes de estudio para su homogeneización con el Espacio Europeo de Educación Superior (EEES). El proceso de convergencia de los planes de estudio universitarios realizado entre 2003 y 2010 se apoyó en la elaboración previa de varios libros blancos encargados por ANECA a grupos expertos, informe que en la esfera de los estudios de comunicación abordó de manera integrada las titulaciones clásicas de Periodismo, Comunicación Audiovisual, y Publicidad y Relaciones Públicas (ANECA 2004). A partir del análisis de la situación en España y el resto de países europeos, el Libro Blanco establecía perfiles profesionales a los que cada plan de estudios debería dar respuesta, y proponía una estructura general básica para los planes que cada universidad podría elaborar después en el diseño de su propia propuesta de plan de estudios.

En este trabajo nos planteamos cuál ha sido el resultado de este proceso de adaptación al Espacio Europeo de Educación Superior en lo referente al tratamiento del periodismo especializado, y más concretamente, del periodismo político, en los planes de estudio resultantes. Veremos que esta presencia es notablemente insuficiente y resulta poco coherente con la importancia del periodismo político, especialmente en la prensa escrita.

\section{Método y datos para el estudio}

Creemos que el proceso de convergencia europea, precisa- mente por su simultaneidad con la crisis en que está inmersa la propia profesión, habría sido un momento idóneo para adaptar los estudios a la realidad profesional (Borrat 1993; Humanes 2005; Meneces 2007; Sierra 2010; Prestano et al. 2011). Lamentablemente, el análisis que presentamos muestra que los perfiles profesionales de Periodismo identificados en el Libro Blanco parecen ignorar las competencias esenciales definitorias de la profesión. Esto es especialmente grave en el reflejo de las áreas de especialización del periodismo y, como veremos, se traduce en una significativa ausencia: una gran mayoría de los estudiantes de grado pueden licenciarse sin formación específica en periodismo político, siendo este, sin embargo, el ámbito de especialización de mayor presencia en la prensa diaria (lo mismo podría afirmarse de otras especialidades igualmente importantes, como el periodismo digital, o el periodismo económico).

Así pues, en este estudio valoraremos dos factores:

- En primer lugar, se analizan los perfiles profesionales identificados para Periodismo por el Libro Blanco sobre los títulos de grado en comunicación (ANECA 2004) ${ }^{1}$. $\mathrm{y}$, consecuentemente, el plan de estudios modelo que se deriva de ellos.

- En segundo lugar, se revisa el despliegue de las recomendaciones contenidas en dicho Libro Blanco en la elaboración concreta de los planes de estudio de Grado en Periodismo.

El análisis de la asignatura de Periodismo político de las universidades españolas se ha basado en la identificación completa de esta asignatura en todas las universidades, tanto públicas como privadas. Se ha considerado conveniente, al respecto, esbozar un mapa completo de la situación de la docencia de periodismo político que incluya todos los perfiles posibles de la asignatura (básica, obligatoria, optativa; curso). Por último, se ha identificado, en el caso de no existir la asignatura con tal denominación, los módulos o materias donde se enseña Periodismo especializado al entenderse que el periodismo político podría ser materia docente dentro de esta asignatura.

\section{Análisis y discusión}

3.1. Importancia del periodismo especializado y del periodismo político en la prensa escrita

El periodismo especializado se define como "el que resulta de la aplicación minuciosa de la metodología periodística de investigación a los múltiples ámbitos temáticos que conforman la realidad social, condicionada siempre por el medio de comunicación que se utilice como canal, para dar respuesta a los intereses y necesidades de las nuevas audiencias sectoriales" (Quesada 2012: 24). Junto a la metodología rigurosa y la focalización temática, el periodismo especializado se identifica también porque persigue "explicar en profundidad la sociedad en la que se insertan los medios de comunicación” (Quesada 2001: 129). Quesada deja claro que la metodología del periodismo de investigación es común a todas las manifestaciones del periodismo especializado, y que exige, por parte del informador, una actitud concreta, dirigida a ir más allá del primer punto de vista que nos puede ofrecer el qué de una información. Desde este plan-

${ }^{1}$ Para la coordinación de todas las facultades en el proyecto se creó un grupo de trabajo formado por Javier Davara (UCM), Xosé López (USC) y Miguel Nieto (US), y dirigido por Marcial Murciano (UAB). 
teamiento resulta plausible considerar el periodismo político como periodismo especializado, más aún por la expansión del periodismo digital, que está forzando a la prensa escrita a jugar un papel más interpretativo, es decir, más orientada a explicar el por qué y el cómo de los hechos que el qué de los mismos. De alguna manera, la noticia generalista ha migrado del papel a la web.

Por lo que se refiere a la importancia del periodismo político en la realidad de la prensa diaria, un análisis del contenido de las portadas de los principales diarios nacionales (en concreto, El País, El Mundo y La Vanguardia) muestra que un $49,2 \%$ de las noticias publicadas en las portadas de la prensa escrita es información. Estos datos son resultado de un análisis (Enguix 2013) realizado mediante la metodología de la semana construida ${ }^{2}$, en el que se analizó la esfera temática de las noticias de portada de estos tres diarios durante 2011; para ello se confeccionó una muestra aleatoria y estratificada que dio como resultado 45 portadas de cada uno de los diarios señalados, con un total de 135 portadas, equivalente, según esta metodología, a 362 ediciones de cada uno de estos periódicos, con un total de 1.086 ediciones.

También las investigaciones basadas en encuestas a profesionales (Giró 1995; Reineman 2004; Charron 2006; Rodríguez-Polo y Santillán 2012; Van Dalen 2012; Enguix 2013) ponen de manifiesto la absoluta prioridad que se otorga a esta especialización del periodismo sobre todo en la prensa escrita. Creemos que esta realidad evidencia la necesidad de proveer al futuro profesional de una formación concreta en este campo, formación que en una situación óptima debería ser tanto teórica como práctica.

3.2. La visión del periodismo derivada del Libro Blanco y plasmada en el diseño de los títulos de Grado

Murciano señala que una de las principales directrices generales derivadas del Libro Blanco era "diferenciar los estudios de comunicación de acuerdo con las demandas del mercado en tres titulaciones profesionales y finalistas: graduado en Periodismo, graduado en Comunicación Audiovisual y graduado en Publicidad y Relaciones Públicas" (2010: 7).

Lamentablemente, y pese a que se afirma esta intención de partida, creemos que el Libro Blanco de la ANECA describe para el Plan de Grado en Periodismo una situación escasamente vinculada a la actual realidad profesional; $y$ esto se comprueba de manera especial al ver el modo en que se caracterizan los perfiles profesionales a los que se orienta la titulación, un modo que no dudaríamos en calificar de neutro, incluso aséptico (ANECA 2004: 191). Como se ve en la cita que reproducimos, la premisa subyacente es que la orientación que podría considerarse estrictamente periodística (y la pluralidad de perfiles que incluye) se equipara en importancia con la orientación en comunicación institucional, en investigación/educación, y en redacción/gestión digital:

“1. Redactor/a de información periodística en cualquier tipo de soporte. Profesional del periodismo en cualquier soporte mediático tradicional o electrónico que desarrolla su actividad mediante los géneros creando contenidos periodísticos. Comprende las tareas de redactor, reportero, presentador y director de uno o de varios de ellos, incluido el diseño escritura y ejecución de guiones de reportajes y documentales audiovisuales o multimedia.

2. Redactor/a o responsable de prensa o comunicación institucional. Profesional de un gabinete de prensa o comunicación de una institución pública o privada para coordinar o ejecutar cuantos trabajos de carácter informativo o comunicativo necesite la entidad.

3. Investigador/a, docente y consultor/a de comunicación. Especialista en investigación y análisis de fenómenos y procesos de comunicación para todo tipo de organizaciones públicas y privadas, capacitado para las tareas de asesoría, consultoría y mediación. Docente en las enseñanzas superiores o medias para impartir materias de comunicación y nuevas tecnologías de la información y la comunicación.

4. Gestor/a de portales y editor/a de contenidos. Profesional en la redacción y ejecución de trabajos de edición en general para empresas editoras o creadoras de producciones culturales e informativas. Especialista en el tratamiento, la gestión y la edición de todo tipo de contenidos por medio de sistemas preferentemente digitales."

Esta pluralidad de perfiles profesionales (reproducidos casi literalmente en algunos de los planes de estudio) puede obedecer, como así se justifica en el informe, a la realidad laboral de los egresados de Periodismo, pero parece incongruente con la decisión de mantener las tres titulaciones ${ }^{3}$ tradicionales de Comunicación Audiovisual, Publicidad y Relaciones Públicas, y Periodismo, debido a lo que se refiere como "una empleabilidad cada vez más segmentada de estas titulaciones" (ANECA 2004: 17).

Así, mientras por un lado se afirma que "un periodista requiere conocimientos diferenciados a los de un publicitario y estos a su vez son significativamente diferentes a los que se requieren para trabajar en un entorno de producción audiovisual y multimedia”, los perfiles profesionales propuestos para el Grado en Periodismo carecen de la especificidad que, sin embargo, sí apreciamos en los otros dos grados. Así, los perfiles profesionales que se proponen para el título de Comunicación Audiovisual son (ANECA 2004: 226):

- Director, guionista y realizador audiovisual.

- Productor y gestor audiovisual.

- Diseño de producción y postproducción visual y sonora.

- Investigador, docente y experto en estudios visuales.

Por su parte, para el grado en Publicidad y relaciones públicas, los perfiles profesionales subyacentes al diseño del plan de estudios son (ANECA 2004: 271):

\footnotetext{
${ }^{2}$ Como es sabido, esta metodología (Krippendorff 1990) permite que un mes de noticias pueda quedar representado en una semana, y un año de noticias en 45 días representativos de la semana construida durante todas las semanas del año. Se logra, además, evitar la contaminación que podría suponer, como en caso que referimos, el análisis de portadas en periodos electorales, que suelen recargar de información política los medios de comunicación en general. La metodología de la semana construida evita estas distorsiones y logra mostrar de forma equilibrada la tendencia en la manera en la que destacamos editorialmente las temáticas durante el periodo estudiado.

${ }^{3}$ La evaluación del Libro Blanco realizada por ANECA señalaba de hecho, como debilidad, la continuidad de tres títulos diferenciados: “la estructura del [Libro Blanco] parece indicar que las tres titulaciones han ido progresando en el proyecto por separado sin mostrar las diferencias ni justificar la necesidad de tres títulos diferentes. Se echa de menos la demostración de esta cuestión a partir de una comparación sistemática de los resultados obtenidos en las tres titulaciones".
} 
- Dirección de comunicación y consulta estratégica en publicidad y relaciones públicas.

- Investigación, planificación y compra de medios.

- Creatividad y diseño publicitario en todo tipo de soportes.

- Gestión de comunicación corporativa.

Si comparamos estos perfiles con los ya reseñados para el grado de Periodismo, vemos que los perfiles descritos en 2,3 y 4 son, sin duda, importantes y esenciales en el conglomerado general de la comunicación, pero desde nuestro punto de vista no son exactamente perfiles periodísticos.

A la hora de buscar una posible explicación para esta concepción poco especializada del Graduado en Periodismo, cabe pensar que el Libro Blanco se elabora en un momento en que los puestos de trabajo vinculados al ecosistema comunicativo se cubrían ${ }^{4}$ fundamentalmente con licenciados en Periodismo: "Según los datos [licenciados en el año 2000] desagregados por carreras, dos tercios de los licenciados en estudios del ámbito de la Comunicación lo hicieron en Periodismo, un 18 \% en Comunicación Audiovisual y un $16 \%$ en Publicidad y Relaciones Públicas. Es decir, que los periodistas eran más del triple que los que terminaron los estudios en las otras dos carreras" (ANECA 2004: 147).

Lo que señalamos puede verse más claramente al comparar los perfiles profesionales del Título de Grado en Periodismo del Libro Blanco, con el rol profesional que se maneja en el Plan Modelo de los Estudios de Periodismo publicado por la UNESCO en 2007. En este documento sí encontramos alusiones explícitas a la necesidad de incluir asignaturas de Periodismo político (entre otras), algo que se vincula con la propia manera de concebir el periodismo como práctica profesional: "el objetivo básico de la mayoría de los periodistas es servir a la sociedad informando a la ciudadanía, interrogándose sobre el modo en que se ejerce el poder, favoreciendo el debate democrático y, con ello, contribuyendo al desarrollo político, económico, social y cultural. La enseñanza del periodismo tendría que instruir a los estudiantes acerca de la manera de identificar noticias y reconocer el hecho de interés informativo en un complejo entorno de datos y opiniones, de realizar investigaciones periodísticas, y de escribir, ilustrar, corregir y producir material en diversos formatos de medios de comunicación (periódicos $\mathrm{y}$ revistas, radio $\mathrm{y}$ televisión e Internet $\mathrm{y}$ multimedia) y para el público propio de esos formatos. Tendría que aportarles los conocimientos y la formación necesarios para reflexionar sobre la ética periodística y las mejores prácticas en el periodismo, además de en torno a la función del periodismo en la sociedad, la historia del periodismo, el Derecho periodístico y la economía política de los medios de comunicación (la propiedad, la organización y la competencia, entre otros). Debería enseñarles cómo informar sobre cuestiones políticas y sociales de especial alcance para su propia sociedad" (UNESCO 2007: 7).
Creemos, en definitiva, que la concepción de los perfiles profesionales del Libro Blanco puede explicar la poca atención prestada en los grados a las diversas especializaciones del periodismo y, muy especialmente, al periodismo político. Pese a la intención declarada inicialmente de incluir entre los objetivos del título en Periodismo, la "formación en Teoría, Historia y Estructura de la Información y la Comunicación para conocer los fundamentos y las prácticas más habituales de los procesos de producción, distribución y recepción de la comunicación desde las perspectivas económica, social, cultural y política, de manera que se proporcione una competencia contextual para situar el periodismo en el campo de la comunicación" (ANECA 2004: 309), lo cierto es que la estructura de plan de estudios que se propone no responde a esta intención. Entre las asignaturas sugeridas como esquema global, el documento propone 16 créditos de contenidos comunes, que, bajo la denominación de Periodismo especializado incluyan el "estudio de la producción informativa de diferentes áreas de especialización periodística, así como de las tendencias que desarrolla esta materia con los diferentes soportes, medios y sistemas: político, económico, cultural, de sociedad, deportivo, científico, local, de investigación y precisión, etc." (ANECA 2004: 314).

Dedicar 16 créditos al Periodismo especializado supone un $6,6 \%$ de los 240 créditos de toda la titulación, por lo que creemos que no refleja la importancia profesional de este cuerpo teórico. La especialización periodística se ha consolidado en las últimas décadas como una realidad incuestionable en los diarios, en papel o digitales (Borrat 1989, 1993; de Fontcuberta 2006; Quesada 1998, 2012; Giró 2010), por lo que la formación de los periodistas no solo debería dar respuesta a esta situación, sino que además debería reflejar entre tales especializaciones las que reciben atención prioritaria por parte de la prensa que, como hemos indicado, son de temática política. El diseño de los planes de estudio debería incorporar la pluralidad disciplinar que caracteriza las profesiones relacionadas con la información y comunicación, proporcionando las necesarias bases teóricas, pero también ha de considerar la vertiente estrictamente laboral ${ }^{5}$ del periodismo.

El informe del Libro Blanco, no obstante, sale al paso de esta realidad, negando su importancia y aduciendo las necesidades empresariales de los años 90 como justificación suficiente para no reflejar la importancia de la especialización en los planes de estudio: “...haremos un apunte acerca de la especialización, que varios investigadores (Díaz Nosty, Casasús, Tuñon y otros, citados por Ramírez de la Piscina, 1999) consideran que marcará el futuro de la profesión. Aunque desde los años setenta se han hecho varios estudios en este sentido que muestran esta tendencia, la realidad que han dibujado los profesionales no lo demuestra tan claramente. Además, para las empresas es más rentable tener profesionales generalistas, que especialistas con menos fle-

\footnotetext{
4 “Las cifras globales para el conjunto de España que aporta la encuesta CHEERS (1998) sólo hacen referencia a la licenciatura de Periodismo, pero como son el colectivo más numeroso dentro del ámbito de la Comunicación, cabe comentarlas: cuatro años después de terminar la carrera (datos referidos a 1998), la tasa de actividad de los licenciados en Periodismo era del 90,9\%, dentro de lo cual, un 7,8 \% corresponde la tasa de paro (...). El $9,9 \%$ que faltaría para completar el $100 \%$ representa a los que continuaron estudiando y a los que se retiraron del mercado laboral. Estas cifras sitúan a los periodistas en un nivel medio por lo que se refiere a tasa de actividad y de paro." (ANECA, 2004: 152).

${ }^{5}$ No nos ocupamos en este trabajo de otro aspecto importantísimo de los planes de estudio: la inclusión de créditos prácticos en empresas. Un examen atento de los planes de estudio de grado (Enguix 2013) muestra que 15 de las 37 universidades permiten obtener el título de Grado en Periodismo sin haber realizado prácticas profesionales en empresas (aunque 8 de ellas ofrecen la opción como asignatura optativa, casi siempre de 6 créditos); por el contrario, 22 universidades sí las incluyen como obligatorias.
} 
xibilidad. En este sentido, el profesor Mariano Martínez Sánchez, que realizó una encuesta sobre este tema en 1994, concluía su estudio diciendo que 'los buenos profesionales tendrán que contar con una amplia formación de base, capacidad de comunicación y divulgación de contenidos"” (ANECA, 2004: 161-162).

Efectivamente, el estudio de Ramírez de la Piscina (1999: 265) refiere los resultados de tres encuestas sobre especialización periodística efectuadas en España en 1974 (realizada a 20 directores de periódicos por Pedro Orive y Concha Fagoaga), en 1994 (Mariano Sánchez Martínez, realizada a profesionales de 19 periódicos) y en 1998 (AIPET, Asociación Iberoamericana de Periodistas Especializados y Técnicos, entrevistas a 496 periodistas especializados españoles), y señala que en los tres momentos reflejados en estas encuestas, la especialización periodística era considerada como algo positivo por los profesionales, pero siempre subordinado al equilibrio económico de las empresas.

Este mismo argumento es utilizado en sentido contrario por Turner y Orange (2013) en su Introducción al monográfico Specialist Journalism, cuyo punto de partida es, precisamente, la evidencia de que la realidad actual resulta demasiado complicada para el periodista todoterreno (jack for all trades), pese a que este perfil generalista no se da en otras profesiones cercanas al periodismo. Se señalan también, en este mismo monográfico, algunos riesgos de la especialización más vinculados al desempeño profesional, como el de "becomes too intimate with one's sources and too anxious to protect them" (Knightley 2013: xi).

3.3. La presencia del Periodismo político en los planes de estudio de grado

Frente a otras áreas de conocimiento, donde el proceso de convergencia europea ha propiciado la aparición de titulaciones con denominaciones muy variadas, el actual catálogo de titulaciones de Grado relacionado con nuestra titulación mantiene la denominación clásica (Grado en Periodismo) y ofrece un total 37 Grados en Periodismo, en 36 de las 75 universidades españolas. De estos 37 grados, solo 13 tienen una asignatura específicamente dedicada al periodismo político, que es optativa en ocho de los planes de estudio (Autònoma de Barcelona, Carlos III, Castilla-La Mancha, Jaume I, Málaga, Pompeu Fabra, Valladolid y Vic), y obligatoria en cinco (Camilo José Cela, Sevilla, SevillaEUSA, San Jorge y Valencia):

- Universidad Camilo José Cela, Facultad de Ciencias de la Comunicación: Periodismo Político (módulo Periodismo Especializado, con 3 créditos de P. Digital y 3 créditos de $P$. Económico); OBL; $3^{\circ}$ curso; 6 créditos.

- Universidad San Jorge, Facultad de Ciencias de la Comunicación: Periodismo político; OBL; $4^{\circ}$ curso; 6 créditos.

- Universidad Carlos III, Facultad de Humanidades, Comunicación y Documentación: Periodismo político y parlamentario; OPT; $3^{\circ}$ curso; 6 créditos.

- Universidad de Castilla-La Mancha, Facultad de Periodismo: Periodismo Político ${ }^{6}$; OPT; $4^{\circ}$ curso; 6 créditos. - Universidad de Málaga, Facultad de Comunicación: Pe- riodismo Político y Económico; Laura Teruel Rodríguez (Titular de Universidad); OPT; $3^{\circ}$ curso; 6 créditos. - Universidad de Sevilla, Facultad de Comunicación: Periodismo político y económico; $\mathrm{OBL} ; 3^{\circ}$ curso; 6 créditos. - Universidad de Sevilla, Centro Adscrito EUSA: Periodismo político y económico; OBL; $3^{\circ}$ curso; 6 créditos. - Universidad de Valladolid, Facultad de Filosofía y Letras: Periodismo político; OPT; $4^{\circ}$ curso; 6 créditos. - Universitat Autònoma de Barcelona, Facultat de Ciències de la Comunicació: Periodisme polític i parlamentari (Itinerario en Periodismo Especializado en Política y Economía); OPT; $4^{\circ}$ curso; 6 créditos.

- Universitat de València, Facultat de Filologia, Traducció i Comunicació; Periodismo político y su tratamiento; OBL; $3^{\circ}$ curso; 6 créditos.

- Universitat de Vic, Facultat d'Empresa i Comunicació: Periodismo político (Mención en Periodismo Político, Económico e Institucional); OPT; $3^{\circ}$ curso; 6 créditos. - Universitat Jaume I, Facultat de Ciències Humanes i Socials; Periodismo político y parlamentario; OPT; $4^{\circ}$ curso; 4,5 créditos.

- Universitat Pompeu Fabra, Facultat de Comunicació: Periodismo especializado en política; OPT; $4^{\circ}$ curso; 4 créditos.

No obstante, sí es más habitual la presencia de asignaturas sobre Periodismo especializado, cuyos temarios suelen incluir (aunque no siempre) un tratamiento explícito del ámbito del periodismo político. De hecho, en 22 de los grados aparece la asignatura de Periodismo especializado ${ }^{7}$, gran parte de las veces como asignatura de dos cursos de grado (I y II), como es el caso de la Universitat CEU Abat Oliva, la Universidad Católica San Antonio, o la Universidad CEU San Pablo de Madrid. La definición de la asignatura no siempre es la misma. En general se la denomina Periodismo Especializado, pero en otros casos Introducción al Periodismo Especializado (Universidad Francisco de Vitoria) o Áreas de especialización periodística (Universidade de Santiago de Compostela). Ya en su artículo sobre especialización del periodismo, previo a la implantación del Espacio Europeo de Educación Superior, Borrat señalaba la posibilidad de: "Dos maneras de estructurar los cursos de Periodismo Especializado (PE) como asignatura universitaria (Información Periodistica Especializada): la que hace teoría general del PE y la que se concentra en alguna de sus manifestaciones específicas" (Borrat 1993: 79).

Hay universidades, como la de Castilla-La Mancha, la Autònoma de Barcelona o la Pompeu Fabra, con itinerarios específicos de especialización. En otras coexiste la formación en Periodismo especializado con asignaturas de subespecialización obligatorias y optativas, reflejando las dos mismas posibilidades que mencionaba Borrat en 1993. En otros casos vemos que no aparecen materias de Periodismo especializado, pero sí asignaturas concretas que proporcionan a los estudiantes formación específica en áreas y metodologías de especialización, por ejemplo:

- Universidad a Distancia de Madrid: optativas de 3 créditos en Periodismo deportivo, Periodismo económico,

\footnotetext{
${ }^{6}$ La asignatura aún no se ha implantado, y la UCLM ha solicitado sustituirla por "Periodismo Internacional".

${ }^{7}$ Las directrices generales de la Licenciatura en Periodismo, establecidas en el RD.1428/1991, incluían una asignatura troncal de Periodismo especializado, con un total de 12 créditos (4 teóricos y 8 prácticos).
} 
Periodismo cultural, Periodismo judicial, y Periodismo de precisión.

- Universidad Camilo José Cela: obligatorias de 3 créditos: Periodismo deportivo, Periodismo político, Periodismo económico, Periodismo científico $y$ medioambiental. Otra optativa en Periodismo de investigación.

- Universidad Carlos III de Madrid: obligatorias de 6 créditos: Información periodística sobre situaciones, tendencias y problemas sociales, Periodismo de proximidad, Periodismo internacional I y II, Periodismo científico y medioambiental. Optativas de 3 créditos: Información de sucesos y tribunales, Periodismo político y parlamentario, Periodismo deportivo.

- Universidad Complutense: obligatorias de 6 créditos: Periodismo especializado en ciencia y cultura, Periodismo especializado en educación y deporte, Periodismo especializado en economía y medio ambiente.

- Universitat de València: obligatorias de 6 créditos en Periodismo de sociedad y cultura, Periodismo económico y su tratamiento, Periodismo político y su tratamiento, Periodismo local y comarcal. Optativas de 6 créditos en Periodismo de ciencia y tecnología y su tratamiento, y de 4,5 créditos en Periodismo de conflictos y de paz, Periodismo cinematográfico, Periodismo deportivo.

El otro modelo frecuente es que exista la obligatoriedad de Periodismo especializado, y algunas optativas que permitan profundizar en esa especialización:

- Universidad de Castilla-La Mancha: una obligatoria en Periodismo especializado. Optativas del itinerario en Periodismo especializado: Periodismo cultural, Periodismo local, Periodismo económico, Periodismo científico, Periodismo deportivo, Periodismo político.

- Universitat Jaume I: obligatoria de Periodismo especializado (6 créditos), y optativas de 4,5 créditos: Periodismo económico, Periodismo político y parlamentario, Periodismo cultural, Periodismo deportivo.

- Universidad de La Laguna: obligatoria de Periodismo especializado (6 créditos), y optativas de 6 créditos en Comunicación biomédica, tecnológica y ambiental, Periodismo turístico y de viajes, Periodismo de proximidad de información local, Comunicación y educación. - Universidad de Málaga: obligatoria de 6 créditos en Teoría y práctica del periodismo especializado, y optativas de 6 créditos en Periodismo y construcción europea, Periodismo político y económico, Periodismo ciudadano $y$ redes sociales.

Otro dato interesante es que en 21 de los 37 grados de periodismo analizados aparece la asignatura de Comunicación Política, bien como Comunicación política estricta, o bien con otros enfoques, como Gestión creativa de la Comunicación Política (U. Camilo José Cela), Comunicación política y procesos electorales (U. Católica San Antonio), Opinión pública y comunicación política (CEU San Pablo), Información y comunicación política (Complutense de Madrid). En doce de estos grados no existe Periodismo Político, y en ocho de los Grados Comunicación Política coexiste como asignatura junto a Periodismo Político. Las Universidades que incluyen en sus planes de Grado los tres tipos de asignatura (Periodismo Especializado, Periodismo Político y Comunicación Política) son cinco: Universidad a Distancia de Madrid, Universidad de Castilla-La Mancha, Universidad de Valladolid, Universitat Autònoma de Barcelona, y Universitat Jaume I. Es decir, los Grados de Periodismo dan globalmente una presencia mayor a la asignatura de Comunicación Política que la asignatura de Periodismo Político, lo que parece otorgar prioridad a orientaciones profesionales (DirCom) no estrictamente periodísticas, sino más propias de los asesores y directores de comunicación de empresas o instituciones.

Otro dato significativo es que la asignatura de Periodismo Político sólo es obligatoria en cinco de los trece Grados en que aparece. No obstante, sí suele tener valor obligatorio la asignatura de Periodismo Especializado, que en ocho universidades constituye el único espacio donde abordar las temáticas de especialización (Nebrija, CEU Abat Oliva, La Laguna, País Vasco, Francisco de Vitoria, Tecnología y Empresa, Santiago de Compostela, e Illes Balears).

Para terminar de comentar la relación entre la asignatura de Periodismo político y las demás asignaturas de cada plan de estudios, señalaremos que gran parte de las universidades ofrecen, generalmente en los cursos de materias básicas, asignaturas específicas relacionadas con otras áreas temáticas que son de necesario conocimiento para el profesional del área. Por ejemplo, el Plan de estudios de la Universitat de València ejemplifica este extremo, ya que junto a la asignatura optativa de Comunicación política que hemos reflejado en el cuadro anterior (cuyo nombre exacto es Comunicación política y opinión pública), los estudiantes cursan diversas materias que les aportan conocimientos esenciales para el periodismo político. En concreto, las asignaturas básicas (todas de 6 créditos) de:

\section{- Historia del mundo actual \\ - Derecho de la comunicación \\ - Sociedad actual \\ - Introducción a la economía actual \\ - Instituciones políticas contemporáneas}

Y además, asignaturas obligatorias de:

- Periodismo de conflictos y paz

- Teoría e historia del periodismo I, y II

Así como asignaturas optativas de:

- Comunicación política y opinión pública

- Geografía del mundo actual

Esta atención a las necesidades del periodismo/periodista político, se reflejan igualmente en la relación de competencias específicas y genéricas inventariadas en el Plan de estudios. Situaciones similares encontramos en otros grados, con diversas asignaturas relacionadas con los temas políticos, incluso itinerarios completos. Por ejemplo, la U. Autònoma de Barcelona tiene un itinerario o mención en Periodismo Especializado en Política y Economía, la Universidad CEU San Pablo ofrece un doble grado en Ciencias Políticas y Periodismo, y la Universidad Pompeu Fabra tiene un itinerario en Ciencias Políticas.

También es frecuente que existan otras asignaturas donde se abordan las temáticas propias del periodismo político, como ya ocurría en las licenciaturas previas; así, encontramos asignaturas de Relaciones internacionales (U. 
Zaragoza, U. Europea Miguel de Cervantes), Sistema Político Español (CEU San Pablo), Ciencia Política y Relaciones Internacionales e Historia del Pensamiento Político Contemporáneo (Complutense de Madrid), Instituciones Políticas Contemporáneas (U. Castilla-La Mancha), Ciencia Política (U. Málaga), Ciencia Política y Opinión Pública (U. Murcia) y otras.

Una vez identificado este panorama general, donde resulta evidente la escasa presencia del Periodismo político en la formación de los futuros periodistas españoles, cabe plantearse si la disminución de créditos que supuso la conversión de Licenciaturas en Grados europeos había afectado especialmente a nuestro ámbito temático. Así, contrastamos los planes de grado con los de las licenciaturas en los años 90, y comprobamos que en general ha habido una reducción del peso del periodismo especializado en los planes de estudio.

Como sabemos, los planes de Licenciatura tenían unas directrices generales de ámbito estatal donde se fijaban las asignaturas troncales y la participación de las áreas de conocimiento; además, permitían una proporción de optatividad mayor a la de los grados europeos. Por tanto, mientras en todas las universidades había una materia troncal de 12 créditos de Periodismo especializado ${ }^{8}$, que cada universidad desarrollaba con los contenidos que consideraba apropiados, la existencia de una asignatura sobre Periodismo político sólo era posible entre las obligatorias y optativas propias de cada universidad, coexistiendo con asignaturas habitualmente asociadas a otras áreas de troncalidad, como Opinión pública (Sociología, Comunicación audiovisual, Psicología social), Relaciones internacionales (Derecho internacional público y relaciones internacionales), Instituciones políticas contemporáneas (Historia contemporánea, Ciencia política y de la administración), y similares.

El análisis de las licenciaturas muestra un panorama dispar, pues mientras en algunas universidades se observa, efectivamente, que el proceso de adaptación al EEES ha supuesto la desaparición de asignaturas optativas de Periodismo político (así ocurre, por ejemplo, en las universidades de Les Illes Balears, CEU Abat Oliva, Europea Miguel de Cervantes, Sevilla, Vic), en otros casos vemos que las asignaturas optativas se mantienen (U. Carlos III, U. Pompeu Fabra), o bien se han convertido en obligatorias con más créditos (U. València), o bien aparecen como optativas nuevas que no existían en las licenciaturas (U. Málaga, U. San Jorge, U. Autònoma de Barcelona, U. Camilo José Cela, U. Valladolid).

En definitiva, los planes de estudio de Grado no parecen haber sido diseñados en consonancia con la realidad que ofrece el análisis de la prensa española. Si, como hemos visto, el 49,2\% de las noticias de prensa tienen temática política, sería esperable que la formación recibida por los futuros periodistas abordara específicamente las cuestiones relacionadas. No obstante, vemos que es posible terminar el Grado en Periodismo sin haber tenido la asignatura de Periodismo político o incluso de Periodismo especializado, lo que contrasta con las prioridades de la prensa escrita y con el criterio mayoritario reflejado en las encuestas a los profesionales del periodismo en periódicos españoles (Enguix 2013).

\section{Conclusiones}

En este trabajo hemos intentado revisar la adecuación de los planes de estudio de Grado en Periodismo con la necesaria formación en contenidos especializados que exige el ejercicio real de la profesión. En concreto, vemos que el actual diseño de la mayoría de los planes de estudio de los grados de Periodismo en España no da respuesta a la importancia del periodismo político, tanto por la relevancia que tiene en la prensa escrita, como por el carácter fundamental que le otorgan los profesionales del periodismo. Una realidad que se aleja además de las directrices que sí establece, como ejemplo, la UNESCO, y por último, del propio ejercicio profesional. No es entendible que en tan pocas facultades de Periodismo esté implantado el Periodismo Político como asignatura, menos aún como asignatura de formación obligatoria, o que se le otorgue tan poco valor en créditos. Creemos, finalmente, que este desequilibrio confirma un cierto alejamiento del diseño de los planes de formación de la mayoría de universidades españolas con Grado en Periodismo, de la praxis real de esta especialización, y que en ello ha tenido especial importancia el condicionamiento derivado de los perfiles profesionales propuestos en el Libro Blanco. Creemos, por último, que esta divergencia entre la formación ofrecida en la universidad y la realidad de la prensa (y no solo en el ámbito del periodismo político, sino también en lo referente a la obligatoriedad de las Prácticas o a otras especialidades, como el periodismo digital), debería ser revisada en el futuro proceso de evaluación y revisión de los Grados.

\section{Bibliografía}

ANECA (2004). Libro Blanco de los Títulos de Grado en Comunicación,

http://www.aneca.es/var/media/150336/libroblanco_comunicacion_def.pdf

Borrat, Héctor (1989). El periódico, actor político. Barcelona: Gustavo Gili.

Borrat, Héctor (1993). Hacia una teoría de la especialización periodística. Anàlisi, 15, pp. 79-84.

Charron, Jean-Marie (2006). Les journalistes politiques: qui sont-ils? Le Temps des médias, 2006/2, $\mathrm{n}^{\circ} 7, \mathrm{p}$. 176-190. DOI: 10.3917/tdm.007.0176

Enguix Oliver, Salvador (2013). Periodismo político en España: de la academia a las portadas de la prensa. Tesis doctoral, Universitat de València.

Fogel, Jean François y Patiño, Bruno (2005). La Prensa sin Gutemberg. El periodismo en la era digital. Madrid: Punto de lectura, 2007.

Fontcuberta, Mar de (2006). El temario periodístico. En Fontcuberta, Mar de \& Borrat, Héctor (2006). Periódicos: sistemas complejos, narradores en interacción. Buenos Aires: La Crujía Ediciones, pp.55-87.

Giró, Xavier (1995). La relació premsa-política des del punt de vista dels periodistas. Enquesta als periodistes de Política dels mitjans de comunicació de Catalunya. Capçalera, 61.

Giró, Xavier (2010): Discursos y grietas en política: La lógica de los actores, los limites de los medios y las metas periodísticas. En Camacho, Idoia (Coord.): $L a$ especialización en Periodismo. Sevilla/Zamora: Comunicación Social, 2010. pp.75-95.

\footnotetext{
${ }^{8}$ Descriptor oficial en BOE: “Teoría, procesos y técnicas de la información especializada, desde los ámbitos más cercanos al especialista hasta la comunicación colectiva".
} 
Humanes, María Luisa (2005). La enseñanza del periodismo en España. El punto de vista de Le Monde Diplomatique, 3, pp.13-16.

Jones, Janet; Salter, Lee (2012). Digital Journalism. Los Angeles: Sage Pubs.

Kawamoto, Kevin (Ed.) (2003). Digital Journalism. Emerging Media and the Changing Horizon of Journalism. Rowman \& Littlefield Publishers.

Knightley, Philipp (2013). Foreword. En Barry Turner y Richard Orange (Eds): Specialist Journalism, Nueva York: Routledge, pp. xi-xiii.

Krippendorff, Klaus (1990). Metodología de análisis de contenido. Teoría y Práctica. Barcelona: Paidós Comunicación.

Meneses, María (2007). En torno al periodismo especializado. Consensos y disensos conceptuales. Anàlisi 35, pp. 137-152.

Murciano, Marcial (2010). La transformación de los estudios de comunicación en España. Diálogos de la comunicación 79, pp. 1-10.

Prestano Rodríguez et al. (2011). Transformaciones en los modelos de formación de periodistas en España. El reto europeo. Estudios sobre el Mensaje Periodístico, 17, pp.401-415.

Quesada, Montserrat (2012). Curso de Periodismo Especializado. Madrid: Síntesis.

Quesada, Montserrat (1998). Periodismo especializado. Madrid: Ediciones Internacionales Universitarias.

Quesada, Montserrat (2001). Periodismo Especializado. En Galdón, Gabriel (coord.). Introducción a la comunicación y la información. Barcelona: Ariel, pp. 123138.
Ramírez de la Piscina, Txema (1999). Realidad y utopía de la especialización en periodismo. Zer, 6/4, pp. 261279.

http://www.ehu.es/zer/es/hemeroteca/articulo/realid ad-y-utopia-de-la-especializacion-en-el-periodismo $/ 85$

Reinemann, Carsten (2004): Routine Reliance Revisited: Exploring Media Importance for German Political Journalists. Journalism and Mass Communication Quarterly 81/4, pp. 857-876. http://dx.doi.org/10.1177/107769900408100409. Rodríguez-Polo, Xosé Ramón y Santillán Buelna, José Ramón (2012). Organización y rutinas del periodismo político en la prensa española de referencia. Observatorio Journal 6/4, pp. 215-234.

Sierra Sánchez, Javier (2010). Competencias profesionales y empleo en el futuro periodista: el caso de los estudiantes de periodismo de la Universidad San Pablo CEU. Icono 14, pp.156-175.

Turner, Barry; Orange, Richard (Eds.) (2013): Specialist Journalism, Nueva York: Routledge.

UNESCO (2007): Plan Modelo de Estudios de Periodismo, http://unesdoc.unesco.org/images/0015/001512/151209s.pdf

Van Dalen, Arjen (2012): Structural Bias in Cross-National Perspective: How Political Systems and Journalism Cultures Influence Government Dominance in the News. The International Journal of Press/Politics 17(1): 32-55. DOI: 10.1177/1940161211411087.

| Cita recomendada de este artículo

Enguix Oliver, Salvador (2013). El proceso de Convergencia Europea y el periodismo especializado: el caso del periodismo político. @tic. revista d'innovació educativa. ( $\left.\mathrm{n}^{\circ} 10\right)$. URL. Fecha de consulta, dd/mm/aaaa. 